\title{
CARACTERÍSTICAS CLÍNICAS Y TOXICIDAD DEL TRATAMIENTO DE TUBERCULOSIS EN PACIENTES CON CÁNCER
}

\author{
Luis Cuéllar ${ }^{1, a}$, Carlos A. Castañeda ${ }^{1, b}$, Katerin Rojas ${ }^{1, \mathrm{c}}$, Claudio Flores ${ }^{2, \mathrm{~d}}$, Ketty Dolores-Cerna ${ }^{1, \mathrm{e}}$, \\ Miluska Castillo ${ }^{1, \mathrm{f}}$, William Vicente ${ }^{1, \mathrm{~g}}$.
}

\begin{abstract}
RESUMEN
Objetivos. Evaluar las características clínico-epidemiológicas de la tuberculosis activa en pacientes portadores de neoplasia maligna y evaluar la influencia del tratamiento antituberculoso sobre el manejo de cáncer en el Instituto Nacional de Enfermedades Neoplásicas entre el 2008 y 2013. Materiales y métodos. Estudio observacional de casos de tuberculosis diagnosticados mediante baciloscopía positiva en pacientes con cáncer. Se revisó la información clínica, evolución, e información patológica de la neoplasia. Resultados. Se encontraron 76 casos de tuberculosis activa luego del diagnóstico de cáncer. La mediana de edad fue 51,3 años. La mediana de seguimiento fue 2,1 años. Las neoplasias más frecuentes fueron leucemia linfática aguda $(14,5 \%)$ para las hematológicas y cáncer de cérvix $(14,5 \%)$, mama $(10,5 \%)$ y gástrico $(7,9 \%)$ para las no hematológicas. El $27,6 \%$ de los pacientes presentó recurrencia de la neoplasia; el diagnóstico de tuberculosis confundió el estadiaje inicial en 6,9\% y se planteó inicialmente como recurrencia del cáncer en 11,1\% (neoplasias de mama y colon). El diagnóstico de tuberculosis retrasó o influyó en la reducción de dosis del tratamiento antineoplásico en $11,1 \%$ de los casos (leucemia linfática aguda y linfoma no Hodgkin). El 8,3\% de los pacientes presentó toxicidad al tratamiento antituberculoso. Conclusiones. Los pacientes con cáncer pueden presentar infección activa por tuberculosis. El efecto de interferencia del diagnóstico y tratamiento de tuberculosis sobre la evaluación del cáncer y el tratamiento antineoplásico en nuestra serie es mínimo.
\end{abstract}

Palabras clave: Tuberculosis; Cáncer; Toxicidad; Tratamiento (fuente: DeCS BIREME).

\section{CLINICAL FEATURES AND TOXICITY OF TUBERCULOSIS TREATMENT IN PATIENTS WITH CANCER}

\begin{abstract}
Objectives. To assess the clinical and epidemiological characteristics of active tuberculosis in patients with malignancy and to assess the influence of TB treatment on cancer management at the National Institute of Neoplastic Diseases from 2008 to 2013. Materials and methods. Observational study of TB cases diagnosed by positive sputum microscopy in patients with cancer. Clinical information, evolution, and pathologic information of neoplasia was reviewed. Results.76 cases of active tuberculosis after being diagnosed with cancer were found. The median age was 51.3 years. Median follow-up was 2.1 years. The most common cancers were acute lymphocytic leukemia $(14.5 \%)$, for the hematologic cancers; and cancer of the cervix $(14.5 \%)$, breast $(10.5 \%)$, and gastric $(7.9 \%)$ for non-hematological cancers. $27.6 \%$ of patients had recurrence of the tumor; TB diagnosis confounded the initial staging by $6.9 \%$ and was initially stated as cancer recurrence in $11.1 \%$ (breast and colon cancers). The diagnosis of tuberculosis delayed or influenced the dose reduction of the antineoplastic treatment in $11.1 \%$ of the cases (acute lymphocytic leukemia and non-Hodgkin lymphoma). $8.3 \%$ of patients had toxicity to the TB treatment. Conclusions. Cancer patients may have active tuberculosis infection. The interference effect of diagnosis and treatment of tuberculosis on the assessment of cancer and cancer treatment in our series is minimal.
\end{abstract}

Key words: Tuberculosis; Cancer; Toxicity; Treatment (source: MeSH NLM).

\section{INTRODUCCIÓN}

La tuberculosis (TB) es un problema mundial de salud y representa la principal causa de muerte por enfermedades infecciosas curables en el mundo (1). En el Perú se reportaron 113 casos por 100000 habitantes en el año $2009{ }^{(2)}$ la cual es una incidencia alta.

El cáncer también es una enfermedad endémica en nuestro medio y comparte algunas características

\footnotetext{
Instituto Nacional de Enfermedades Neoplásicas. Lima, Perú.

Oncosalud/AUNA. Lima, Perú.

Médico especialista en Enfermedades Infecciosas y Tropicales; ${ }^{\text {b }}$ oncólogo clínico, magíster en Genética y Biología Celular, magíster en Oncología Avanzada; oncólogo clínico; ${ }^{\mathrm{d}}$ licenciado en estadística, magíster en Investigación Operativa; ${ }^{\mathrm{e}}$ bioestadístico; ${ }^{\mathrm{f}}$ toxicóloga; ${ }^{\mathrm{g}}$ médico patólogo

Recibido: : 06-10-2014 Aprobado: 22-04-15
}

Citar como: Cuéllar L, Castañeda CA, Rojas K, Flores C, Dolores-Cerna K, Castillo M, et al. Características clínicas y toxicidad del tratamiento de tuberculosis en pacientes con cáncer. Rev Peru Med Exp Salud Publica. 2015;32(2):272-7. 
clínicas y procesos fisiopatológicos con la TB. Ambos procesos se asocian a cuadros de inflamación crónica sistémica y a alteraciones en la respuesta inmune (3). Algunas series sugieren que el diagnóstico del cáncer y la administración de quimioterapia son factores de riesgo para el desarrollo de TB. La asociación TB y cáncer ha sido descrita con mayor frecuencia en el cáncer de pulmón, cáncer de cabeza y cuello, y malignidades linfoproliferativas ${ }^{(4-9)}$.

La TB puede ocasionar sintomatología e inclusive producir lesiones que, en algunos casos, se pueden confundir con lesiones neoplásicas y así alterar el enfoque y tratamiento antineoplásico. La TB produce un cuadro sistémico de desnutrición y consumo que puede influir la tolerancia a los tratamientos antineoplásicos. El tratamiento antituberculoso consta de la combinación de agentes altamente tóxicos que pueden alterar el estado general del paciente oncológico y la tolerancia a drogas antineoplásicas, así como también interaccionar con los agentes antineoplásicos. Igualmente, la asociación con TB concurrente implica un peor pronóstico en los pacientes oncológicos. Algunas series sugieren que la eficacia del tratamiento antituberculoso en pacientes con diagnóstico de cáncer es igualmente efectiva ${ }^{(4,10)}$.

El objetivo del presente trabajo es evaluar las características clínico-epidemiológicas de la TB activa en pacientes portadores de cáncer; así como evaluar la influencia del tratamiento antituberculoso sobre el manejo de cáncer en el Instituto Nacional de Enfermedades Neoplásicas (INEN) en el periodo 20082013.

\section{MATERIALES Y MÉTODOS}

\section{DISEÑO Y POBLACIÓN DEL ESTUDIO}

Se ha revisado en forma retrospectiva todos los casos de TB diagnosticados microbiológicamente mediante baciloscopía positiva en pacientes con diagnóstico de cáncer atendidos en el INEN desde enero 2008 hasta diciembre 2013.

\section{PROCEDIMIENTOS}

El diagnóstico de TB fue realizado en muestras de esputo y orina, así como en tejidos biopsiados mediante visión directa con coloración Ziehl Neelsen. Los cultivos se realizaron mediante los medios de LowesteinJenssen, sin embargo, este estudio fue realizado en la mayoría de casos en el centro de salud cercano al domicilio del paciente y no en el INEN debido a la estrategia política del Programa Nacional de Control de
TB (no contamos con esta información en el documento fuente del Instituto-Historia Clínica).

Así mismo, se revisó y se recabó información clínicoepidemiológica de las historias clínicas. Esta información incluyó datos sobre antecedentes e historia de contacto $\mathrm{TB}$, historia clínica de ingreso, datos anatomopatológicos de la neoplasia, y evolución de los pacientes.

\section{ANÁLISIS ESTADÍSTICO}

La información fue analizada mediante el software estadístico Stata versión 13 para Windows. Para describir a las variables cuantitativas se empleó las medidas de tendencia central y dispersión correspondientes. Además, se utilizó la prueba de chi cuadrado o la exacta de Fisher para determinar la asociación entre variables categóricas. Para establecer la diferencia entre dos proporciones de muestras independientes se empleó la prueba Z. La significancia estadística fue determinada por un valor $p<0,05$.

El tiempo de seguimiento para estimar la sobrevida global comprende desde la fecha de diagnóstico patológico hasta la fecha de muerte o último control; la sobrevida libre de progresión comprende desde la fecha de diagnóstico patológico hasta la fecha de recurrencia o último control. Las curvas de sobrevida se calcularon por el método de Kaplan-Meier y la comparación entre curvas de diferentes categorías de las características se probó mediante log-rank test. Se consideró una diferencia significativa al valor de $p<0,05$.

\section{CONSIDERACIONES ÉTICAS}

El desarrollo de este proyecto no involucró contacto ni intervención sobre los pacientes (estudio observacional de revisión de información descrita en historia clínica). La base de datos creada no incluyó la identidad de las personas y la información obtenida se mantuvo en completa confidencialidad. Este proyecto fue aprobado por el Comité Revisor de Protocolo y el Comité de Ética del INEN.

\section{RESULTADOS}

Se encontraron 76 casos de TB en estado activo luego que los pacientes fueron ingresados con diagnóstico de cáncer al INEN. La mediana de edad fue 51,3 años y la relación hombre-mujer fue de 0,73. Tres casos tuvieron el antecedente de ser VIH-positivos y un caso de síndrome de Down. La mediana del intervalo entre el diagnóstico de cáncer y TB fue de 10 meses (rango: 0-234 meses). 
Tabla 1. Características clínicas de los pacientes con diagnóstico de tuberculosis y cáncer atendidos en el Instituto Nacional de Enfermedades Neoplásicas, 2008-2013

\begin{tabular}{|c|c|c|}
\hline Características clínicas & $\mathbf{N}$ & (\%) \\
\hline Total & 76 & \\
\hline Edad mediana (rango) & $51,3 \mathrm{a}$ & ,2-86,5) \\
\hline \multicolumn{3}{|l|}{ Neoplasia hematológica } \\
\hline Leucemia linfática aguda & 11 & $(14,5)$ \\
\hline Leucemia mieloide aguda & 2 & $(2,6)$ \\
\hline Linfoma no Hodgkin & 7 & $(9,2)$ \\
\hline linfoma de Hodgkin & 4 & $(5,3)$ \\
\hline \multicolumn{3}{|l|}{ Neoplasia no hematológica } \\
\hline Cérvix & 11 & $(14,5)$ \\
\hline Mama & 8 & $(10,5)$ \\
\hline Estómago & 6 & $(7,9)$ \\
\hline Colon & 4 & $(5,3)$ \\
\hline Vejiga & 3 & $(3,9)$ \\
\hline Próstata & 3 & $(3,9)$ \\
\hline Tiroides & 2 & $(2,6)$ \\
\hline Pulmón & 2 & $(2,6)$ \\
\hline Ano & 2 & $(2,6)$ \\
\hline Riñón & 2 & $(2,6)$ \\
\hline Otros & 9 & $(11,8)$ \\
\hline \multicolumn{3}{|c|}{ Estadio clínico de neoplasia- no leucemia } \\
\hline I & 8 & $(10,0)$ \\
\hline II & 17 & $(23,3)$ \\
\hline III & 21 & $(28,8)$ \\
\hline IV & 14 & $(19,2)$ \\
\hline Desconocido & 3 & \\
\hline \multicolumn{3}{|l|}{ Esquema de tratamiento } \\
\hline QT Neo/Adyuvante & 17 & $(23,6)$ \\
\hline RT Neo/Adyuvante & 10 & $(13,9)$ \\
\hline QT/RT concurrente & 9 & $(12,5)$ \\
\hline Solo hormonoterapia & 3 & $(4,2)$ \\
\hline Esquemas QT de leucemia & 13 & $(18,1)$ \\
\hline CHOP/ABVD & 9 & $(12,5)$ \\
\hline Otros & 4 & $(5,6)$ \\
\hline No recibió tratamiento & 7 & $(9,7)$ \\
\hline Desconocido & 4 & \\
\hline Recurrencia de neoplasia & 21 & $(27,6)$ \\
\hline Fallecimientos & 6 & $(8,8)$ \\
\hline Tasa de supervivencia a los 3 años (TSG) & & $(45,9)$ \\
\hline TSG neoplasias hematológicas & & $(36,8)$ \\
\hline TSG neoplasias no hematológicas & & $(49,1)$ \\
\hline
\end{tabular}

Las neoplasias más frecuentes fueron leucemia linfática aguda $(14,5 \%)$ para las hematológicas, y cáncer de cérvix $(14,5 \%)$, mama $(10,5 \%)$ y gástrico $(7,9 \%)$ para las no hematológicas. El estadio clínico más frecuente al debut del cáncer de cérvix, mama y gástrico fueron de III (45,5\%), II y IV (65\%), III y IV (80\%), respectivamente. El 15,4\% (2/13) de los pacientes con leucemias recibió tratamiento con quimioterapia. El 63,6\% (7/11), 18,2\% (2/11) de los pacientes con cáncer de cérvix recibieron radioterapia sola, quimioterapia y radioterapia, respectivamente. El $87,5 \%$ (7/8) de los pacientes con cáncer de mama recibió quimioterapia. El 20\% (1/5) de los pacientes
Tabla 2. Cuadro clínico y exámenes auxiliares de los pacientes con diagnóstico de tuberculosis y cáncer atendidos en el Instituto Nacional de Enfermedades Neoplásicas, 2008-2013

\begin{tabular}{|c|c|c|}
\hline Cuadro clínico & $n=76$ & $(\%)$ \\
\hline Contacto con TB & 13 & $(17,1)$ \\
\hline \multicolumn{3}{|l|}{ Cuadro clínico } \\
\hline Tos de 15 días & 61 & $(44,2)$ \\
\hline Fiebre & 39 & $(28,3)$ \\
\hline Dolor pleurítico & 15 & $(10,9)$ \\
\hline Diaforesis & 4 & $(2,9)$ \\
\hline Hemoptisis & 3 & $(2,2)$ \\
\hline Hiporexia & 3 & $(2,2)$ \\
\hline Dolor lumbar & 2 & $(1,5)$ \\
\hline Hematuria & 2 & $(1,5)$ \\
\hline Fistula esternal & 1 & $(0,7)$ \\
\hline Ninguno & 8 & $(5,8)$ \\
\hline \multicolumn{3}{|l|}{ Cultivo } \\
\hline No se hizo & 43 & $(58,1)$ \\
\hline Negativo & 23 & $(31,1)$ \\
\hline Positivo & 8 & $(10,8)$ \\
\hline Desconocido & 2 & \\
\hline \multicolumn{3}{|l|}{ Localización de TB } \\
\hline Pulmonar & 61 & $(80,3)$ \\
\hline Extrapulmonar & 15 & $(19,7)$ \\
\hline Genitourinaria & 11 & \\
\hline Ganglionar & 1 & \\
\hline Ósea & 1 & \\
\hline Otras & 2 & \\
\hline $\mathrm{RX}$ de tórax: con lesión TB & 57 & $(75,0)$ \\
\hline Granuloma & 8 & $(10,5)$ \\
\hline \multicolumn{3}{|l|}{ Muestra de estudio Bk } \\
\hline Esputo & 49 & $(64,5)$ \\
\hline Orina (incluye esputo) & 13 & $(17,1)$ \\
\hline LBA & 7 & $(9,2)$ \\
\hline Pieza quirúrgica & 3 & $(3,9)$ \\
\hline Líquido pleural & 1 & $(1,3)$ \\
\hline Heces & 1 & $(1,3)$ \\
\hline Piel & 1 & $(1,3)$ \\
\hline Ganglios & 1 & $(1,3)$ \\
\hline
\end{tabular}

con cáncer gástrico recibió quimioterapia. Finalmente, los dos pacientes (100\%) con cáncer de pulmón recibieron radioterapia con o sin quimioterapia. En el $9,7 \%$ de los casos no se administró ningún tratamiento antineoplásico (Tabla 1).

Los síntomas más frecuentes asociados a TB fueron tos de 15 días y fiebre. No existió diferencia en presentación de síntomas entre las neoplasias hematológicas y las no hematológicas $(p=0,685)$. Se encontró alteración radiográfica sugestiva de TB en $75 \%$. El paciente indicó contacto TB en $17,11 \%$ de los casos. Los estudios de baciloscopía mediante Ziehl Neelsen se realizaron en todos los casos y se obtuvieron de muestras de esputo $(64,5 \%)$, orina $(17,1 \%)$, lavado bronquial $(9,2 \%)$ o pieza quirúrgica $(3,9 \%)$. El estudio de tipificación con cultivo se realizó en el Instituto en solo 31 casos, siendo positivo en $10,8 \%$ de los casos (Tabla 2 ). 
Tabla 3. Confusión con estadiaje y recurrencia, e influencia en el tratamiento de los pacientes con diagnóstico de tuberculosis y cáncer atendidos en el Instituto Nacional de Enfermedades Neoplásicas, 2008-2013

\begin{tabular}{lcc}
\hline $\begin{array}{c}\text { Influencia de TB en estadiaje recurrencia y } \\
\text { tratamiento }\end{array}$ & N & (\%) \\
\hline Pacientes & 76 & \\
\hline TB interpretada inicialmente como lesión neoplásica & \\
\hline No produjo confusión & 59 & $(81,9)$ \\
Confundió estadiaje & 5 & $(6,9)$ \\
$\quad$ Confundió recurrencia & 8 & $(11,1)$ \\
Desconocido & 4 & \\
TB influyo el tratamiento de la neoplasia & & \\
No influye & 64 & $(88,9)$ \\
Retraso de tratamiento & 8 & $(11,1)$ \\
Desconocido & 4 & \\
\hline
\end{tabular}

El $27,6 \%$ de los pacientes presentaron recurrencia. El diagnóstico de TB confundió el estadiaje inicial en $6,9 \%$ y se interpretó inicialmente como una posible recurrencia de la neoplasia en $11,1 \%$ de los casos. Este fenómeno de confusión fue más frecuente en las neoplasias de mama y colon. El diagnóstico de TB influyó en el retraso o reducción de intensidad de dosis del tratamiento antineoplásico en $11,1 \%$ de los casos, fue más frecuente en neoplasias hematológicas que en no hematológicas ( 33,3 vs $3,7 \%, p=0,001$ ), en especial en leucemia linfática aguda $36,4 \%(4 / 11)$ y en linfoma no Hodgkin (LNH) 14,29\% (1/7). A pesar de la muestra pequeña, los pacientes con $\mathrm{LNH}$ que sufrieron alteración en intensidad de dosis tuvieron una menor sobrevida libre de enfermedad $(p=0,045)$ (Tabla 3).

El $75 \%$ de los pacientes recibió esquema I de tratamiento anti-TB (rifampicina, isoniacida, pirazinamida y etambutol por un total de 6 meses), el 14,47\% recibió esquema II (rifampicina, isoniacida, pirazinamida, etambutol y estreptomicina por un total de 8 meses) y $9,21 \%$ no recibió ningún tratamiento. El $89,2 \%$ de los pacientes no presentó toxicidad al tratamiento anti-TB y el $4,05 \%$ presentó toxicidad hepática, el $1,35 \%$ toxicidad dérmica, gastritis, insuficiencia renal aguda o purpura trombocitopénica idiopática. Sin embargo, ninguna de estas toxicidades produjo muerte (Tabla 4).

El control baciloscópico luego del tratamiento anti-TB fue realizado en 50 casos, siendo negativo en todos ellos. No se encontró reactivación de tuberculosis.

La mediana de seguimiento fue 2,67 años, se encontró recurrencia de cáncer en 21 casos $(27,63 \%)$, y muerte en $6(7,89 \%)$, todas asociadas al cáncer; la mediana de periodo libre de progresión fue de 24 meses, y aunque la mediana de sobrevida global no fue alcanzada, la sobrevida a los 3 años fue de 45,9\% (Tabla 1).
Tabla 4. Tratamiento antituberculoso y toxicidad al tratamiento de los pacientes con diagnóstico de tuberculosis y cáncer atendidos en el Instituto Nacional de Enfermedades Neoplásicas, 2008-2013

\begin{tabular}{lcc}
\hline Tratamiento y toxicidad & N & (\%) \\
\hline Pacientes & 76 & \\
Esquema & & \\
$\quad$ Anti-TB I & 57 & $(75,0)$ \\
\hline Anti-TB II & 11 & $(14,5)$ \\
\hline No recibió tratamiento & 7 & $(9,2)$ \\
\hline Esquema individualizado & 1 & $(1,3)$ \\
Toxicidad al tratamiento Anti-TB & & \\
\hline Ninguna & 66 & $(89,2)$ \\
\hline Hepática & 3 & $(4,1)$ \\
\hline Rash dérmico & 1 & $(1,4)$ \\
\hline Gastritis & 1 & $(1,4)$ \\
\hline Insuficiencia renal & 1 & $(1,4)$ \\
\hline Purpura trombocitopénica idiopática & 1 & $(1,4)$ \\
\hline Neuropatía & 1 & $(1,4)$ \\
\hline Desconocido & 4 & \\
\hline
\end{tabular}

\section{DISCUSIÓN}

La serie presentada en este trabajo es única debido a que tiene dos características propias de nuestra población y diferente de poblaciones de otras latitudes. La primera característica es nuestra alta prevalencia de cáncer de cérvix y cáncer gástrico en los pacientes que acuden a los centros de salud públicos. La segunda característica es la alta prevalencia de TB en nuestro medio (2). Cuéllar et al. estudiaron 150 casos de cáncer en el INEN y encontraron que $10(6,8 \%)$ pacientes tuvieron antecedente de TB ${ }^{(11)}$. Por otro lado, el mismo investigador reportó una cohorte de 819 casos de cáncer y encontró que el 1,2\% tuvieron coinfección $\mathrm{VIH} / \mathrm{TB}{ }^{(12)}$. Finalmente, esta serie encuentra 76 casos de baciloscopía TB positiva a partir del registro de laboratorio entre los años 2008 a 2013. Durante estos años acudieron 88225 pacientes nuevos al INEN, lo cual produce una tasa de presentación de TB activa en cáncer de 90 por 100000 . Sin embargo, esta tasa tiene severas limitaciones debido a que muchos pacientes realizan el estudio y tratamiento de TB en otra institución y otros se pierden de vista luego del diagnóstico de cáncer.

En este estudio se encontró, en forma similar a otros reportes, que la mayoría de los casos de TB hallados en el Instituto fueron catalogados como pulmonares $(80,3 \%)$. El estudio diagnóstico inicial en todos los casos de este trabajo fue la baciloscopía mediante Ziehl Neelsen. Esta prueba diagnóstica, aunque tienen sensibilidad y 
especificidad baja, es la más utilizada en todo el mundo y en nuestro medio y se realizó satisfactoriamente en la mayoría de casos en muestras de esputo $(64,5 \%)$, aunque en el $17,1 \%, 9,2 \%$ y $3,9 \%$ se realizó en orina, lavado bronquial y pieza quirúrgica, respectivamente. El método de cultivo se realizó en solo 31 casos debido a dificultades logísticas, siendo positivo en $10,8 \%$ de los casos. Este método tiene una alta sensibilidad (89\%) y especificidad (98\%), y logra identificar el M. tuberculosis en casos no detectados por baciloscopía. Con relación a los síntomas, en forma similar a la bibliografía, se encontró que los más frecuentes fueron tos $(80,2 \%)$ y fiebre $(51,3 \%)$. Se encontró lesiones pulmonares compatibles con TB en los estudios de imágenes en el $75 \%$ de los casos. No existieron diferencias significativas por edad o sexo, probablemente por el pequeño tamaño muestral (13-15)

Las leucemias y su tratamiento producen alteraciones en la inmunidad innata y adquirida, estando directamente asociadas con un incremento en infecciones oportunistas, incluyendo TB. Algunos reportes sugieren que leucemias como la leucemia linfática aguda o mieloide aguda tienen mayor predisposición a desarrollar TB activa. En nuestro registro, la neoplasia hematológica más frecuente asociada a coexistencia por TB fue la leucemia linfática aguda. Sin embargo, esta mayor incidencia de asociación puede relacionarse a la prevalencia de la neoplasia, pues todas las leucemias se asocian a una alteración final en la actividad inmune del huésped. La tasa de confusión entre lesiones neoplásicas y TB, al momento del estadiaje y de recurrencia $(18,1 \%)$ fue aceptable, debido a que la endemicidad de TB en nuestro medio obliga a pensar en ella como diagnóstico diferencial a descartar. Sin embargo, frecuentemente existió retraso o reducción de intensidad de dosis en la administración de quimioterapia asociado al diagnóstico y tratamiento de TB, debido a que la entidad infecciosa se asocia a caquexia y desnutrición que dificultan mantener un tratamiento intenso como en neoplasias hematológicas ${ }^{(16,17)}$

En relación a neoplasias sólidas, la asociación entre cáncer de cérvix y TB esté probablemente relacionada a que ambas enfermedades están asociadas con hacinamiento y desnutrición. La coexistencia entre cáncer de mama y TB se asocia al problema clínico que ambas pueden compartir una apariencia similar pudiendo causar confusiones de diagnóstico: la primera frecuentemente se disemina vía linfática a los ganglios cervicales y mediastinales, y vía hematógena a los pulmones; mientras que la TB produce lesiones pulmonares y adenopatías cervicales y mediastinales. La asociación neoplásica más conocida de TB activa es con cáncer broncogénico debido a la colocalización pulmonar y a que ambos cuadros afectan generalmente a adultos mayores. Debido a la posible coexistencia de TB pulmonar y cáncer broncogénico, hay autores que sugieren la posibilidad de realizar broncoscopía y citología de esputo en pacientes tuberculosos con clínica atípica, y examen de esputo y PCR (reacción de cadena polimerasa) en pacientes con cáncer con sintomatología crónica ${ }^{(18)}$.

La posibilidad de que las lesiones por TB sean interpretadas como posibles recurrencias es infrecuente en las neoplasias solidas evaluadas. Así mismo, los casos en que se requirió retrasar o reducir intensidad de dosis del tratamiento antineoplásico debido al diagnóstico o tratamiento de TB fueron escasos aunque más frecuentes en las neoplasias hematológicas, especialmente LNH. Este fenómeno se debió a que la gran mayoría de casos fueron neoplasias sólidas no metastásicas sin indicación de tratamiento sistémico y debido a la buena tolerancia a los tratamientos antineoplásicos. El análisis de sobrevida encontró que los casos con LNH que sufrieron alteración en intensidad de dosis tuvieron una menor sobrevida libre de enfermedad $(p=0,045)$, sin embargo, esta información requiere una análisis en una muestra mayor debido a la presencia de otros factores que influyen el análisis como desnutrición (17)

Sin embargo, la presente serie tiene la debilidad que debido a disposiciones logísticas los pacientes, en la mayoría de los casos, acudieron a otra institución a realizarse el cultivo de $M$. tuberculosis para identificación microbiológica y no se registró este resultado en las historias clínicas del Instituto. La mejoría en los procesos del instituto ha significado un incremento progresivo en el número de cultivos realizados y a la implementación permanente del procedimiento.

Concluimos que nuestros pacientes con cáncer pueden desarrollar TB activa, tanto aquellos con neoplasias hematológicas como leucemia linfática aguda así como con neoplasias sólidas como cáncer de cérvix, mama y gástrico. El efecto de interferencia del diagnóstico de TB en la evaluación del estado de enfermedad de la neoplasia y en el tratamiento antineoplásico en esta serie de casos es mínimo. Recomendamos tener siempre presente el diagnóstico diferencial de coexistencia de infección activa de TB en pacientes con cáncer para así efectivizar un tratamiento infeccioso precoz, evitar interpretación inadecuada de las lesiones causadas por tuberculosis y evitar disminución de intensidad de dosis de tratamiento antineoplásico.

Fuentes de financiamiento: autofinanciado. 
Conflictos de interés: los autores declaran no tener conflictos de interés.

Contribuciones de autoría: CAC, KRL, WV y LC participaron en la concepción y diseño del artículo; KRL, CAC, MCG y WV participaron en la recolección y obtención de resultados; $\mathrm{CF}$,
CAC, KRL, LC y KDC participo en el Análisis e interpretación de datos; CAC, LC, KRL y MCG participaron en la redacción del artículo; LCP, CAC y KRL participaron en la revisión crítica del artículo; LC, KRL y CAC realizaron la aprobación de su versión final; KRL obtuvo el financiamiento; CF y KDC participó en la asesoría estadística.

\section{REFERENCIAS BIBLIOGRÁFICAS}

1. Organización Mundial de la Salud. Tuberculosis. Nota descriptiva No104 [Internet]. Ginebra: OMS; 2015 [citado el 15 de abril del 2014]. Disponible en: http://www.who.int/ mediacentre/factsheets/fs104/es/

2. Perú, Ministerio de Salud. Situación de la tuberculosis en el Peru [Internet]. Lima; MINSA; 2011 [citado el 15 de abil de 2014]; Disponible en: http://www. minsa.gob.pe/portada/Especiales/2011/ respiravida/archivos/Ayuda_memoria Lanzamiento_TB.pdf.

3. O'Garra A, Redford PS, McNab FW, Bloom CI, Wilkinson RJ, Berry MP. The immune response in tuberculosis. Annu Rev Immunol. 2013;31:475527. doi: 10.1146/annurevimmunol-032712-095939.

4. Ibrahim EM, Uwaydah A, alMulhim FA, Ibrahim AM, el-Hassan AY. Tuberculosis in patients with malignant disease. Indian $\mathrm{J}$ Cancer. 1989 Jun;26(2):53-7.

5. Tubura E. [Pulmonary tuberculosis in the compromised host--report of the 30th B series of controlled trials of chemotherapy--Cooperative Study Unit of Chemotherapy of Tuberculosis of the National Sanatoria in Japan]. Kekkaku. 1991 Feb;66(2):95-9. [Artìculo en Japones].

6. Dewan PK, Arguin PM, Kiryanova $\mathrm{H}$, Kondroshova NV, Khorosheva TM, Laserson K, et al. Risk factors for death during tuberculosis treatment in Orel, Russia. Int J Tuberc Lung Dis. 2004;8(5): 598-602.

7. Gopalakrishnan P, Miller JE, McLaughlin JS. Pulmonary tuberculosis and coexisting carcinoma: a 10-year experience and review of the literature. Am Surg. 1975 Jul;41(7):405-8.

8. Jain BK, Chandra SS, Narasimhan R, Ananthakrishnan N, Mehta RB.
Coexisting tuberculosis and carcinoma of the colon. Aust N Z J Surg. 1991 Nov;61(11):828-31.

9. Karnak D, Kayacan O, Beder S. Reactivation of pulmonary tuberculosis in malignancy. Tumori. 2002 MayJun;88(3):251-4.

10. Perú, Ministerio de Salud. Tuberculosis en el Perú, informe 2009 [Internet]. Lima: MINSA; 2009. [citado el 15 de abril del 2014 ]. Disponible en: http:// www.minsa.gob.pe

11. Cuellar L, Biminchumo C, Rosales R, Vicente W, Pinto J, Miranda L. Tuberculosis activa en pacientes con cáncer: experiencia del INEN: 1995-2004. X Congreso Peruano de Enfermedades Infecciosas y Tropicales. Sociedad Peruana de Enfermedades Infecciosas y Tropicales. Lima, Perú. 07 de setiembre del 2007.

12. Cuéllar L, Miranda L, Rosales L, Leon J, Flores C. Características epidemiológicas de los pacientes con cáncer y VIH/SIDA INEN 1988-2007. $\mathrm{X}$ Congreso Peruano de Enfermedades Infecciosas y Tropicales. Sociedad Peruana de Enfermedades Infecciosas y Tropicales. Lima, Perú. 07 de setiembre del 2007.

13. Sequeira de Latini MD, Barreda L; Organización Panamericana de la Salud. Manual para el diagnostico bacteriológico de la tuberculosis. Normas y Guía Técnica [Internet]. Washington, DC: OPS; 2008 [citado el 30 de marzo del 2015]. Disponible en: http://www.sld.cu/galerias/pdf/ sitios/tuberculosis/tb-labs-cultivo[2]. pdf.

14. Shi R, Sugawara I. Pathophysiology of Tuberculosis. En: Mahboub H, Mayank G. Tuberculosis - Current Issues in Diagnosis and Management [Internet]. Rijeka: Intech; 2013. p. 127-40 [citado el 30 de marzo del 2015]. Disponible en: http://www.intechopen.com/ books/tuberculosis-current-issues-indiagnosis-and-management

15. Hatta M, Sultan A. Diagnostic Evaluation of Tuberculosis. En: Mahboub H, Mayank G. Tuberculosis - Current Issues in Diagnosis and Management [Internet]. Rijeka: Intech; 2013. p. 153-161 [Citado el 30 de marzo del 2015]. Disponible en: http://www.intechopen.com/ books/tuberculosis-current-issuesin-diagnosis-and-management/ diagnostic-evaluation-of-tuberculosis

16. Chen CY, Sheng WH, Cheng A, Tsay W, Huang SY, Tang JL, et al. Clinical characteristics and outcomes of Mycobacterium tuberculosis disease in adult patients with hematological malignancies. BMC Infect Dis. 2011 Nov 23;11:324. doi: 10.1186/14712334-11-324.

17. Kim DK, Lee SW, Yoo CG, Kim YW, Han SK, Shim YS, et al. Clinical characteristics and treatment responses of tuberculosis in patients with malignancy receiving anticancer chemotherapy. Chest. 2005;128(4):2218-22.

18. Ito K, Fujimori M, Shingu K, Hama Y, Kanai T, Koyama H, et al. Pulmonary tuberculosis in a patient receiving intensive chemotherapy for metastatic breast cancer. Breast J. 2005 JanFeb;11(1):87-8.

Correspondencia: Luis Cuellar Ponce de León Dirección: Av. Angamos Este 2520-Surquillo, Lima-Perú

Teléfono: 993535911

Correoelectrónico:fcuepaz@infonegocio.net.pe 